\title{
Transcatheter closure of interatrial communications with a modified umbrella device
}

\author{
Andrew N Redington, Michael L Rigby
}

\begin{abstract}
Objective-To show the institutional experience of closure of interatrial communications with a modified umbrella device.

Design-Descriptive study of all patients in whom transcatheter umbrella closure of interatrial communications has been attempted.

Setting-A tertiary referral centre.

Patients-16 patients with 17 interatrial communications of various types.

Interventions-Transcatheter placement of a modified Rashkind umbrella occluder.

Results-Two procedural failures due to venous access problems. Two placement failures needing early surgical repair. 13 successful placements in 12 patients.

Conclusions-The modified Rashkind umbrella can successfully be used to close both iatrogenic and naturally occurring interatrial communications, particularly those present after the Fontan procedure.
\end{abstract}

(Br Heart f 1994;72:372-377)

Surgical closure of a naturally occurring atrial septal defect remains one of the commonest open heart operations performed in children. It has a low mortality and a low early morbidity, but it does require a median sternotomy, cardiopulmonary bypass, and several days in hospital. Furthermore, with the increasing use of a fenestrated atrial septal patch in high risk right and left sided heart disease the benefits of avoidance of an open surgical procedure are even more obvious. Transcatheter device placement, if comparable in terms of its mortality and morbidity, offers the possibility of avoiding surgery under these circumstances. The Lock clamshell was designed specifically for this purpose. ${ }^{1}$ The main difference between this device and the Rashkind patent ductus arteriosus umbrella is its double articulation, which results in circumferential apposition around its margins. The clamshell device has been used successfully to occlude naturally occurring atrial septal defects, muscular and postoperative ventricular septal defects, and the interatrial communication after the fenestrated Fontan operation. ${ }^{1-3}$ Unfortunately it is not currently available for clinical use.
We have previously described our modification of the Rashkind ductal umbrella and its use to treat various different intracardiac and extracardiac communications. ${ }^{4}$ In this study we review our total experience of its use in the closure of interatrial communications.

\section{Patients and methods}

Since August 1991 we have attempted to close 17 interatrial communications in 16 patients. Their ages ranged from 5 days to 34 years and their weights ranged from $3 \cdot 1$ to 64 kg. There were 11 patients who had previously undergone a fenestrated Fontan operation three days to 17 weeks previously. This procedure was performed because of a preoperative high risk of a standard Fontan operation-that is, mean pulmonary arterial pressure $>15 \mathrm{~mm} \mathrm{Hg}$, main chamber hypertrophy, or complex atrial and ventricular morphology. Ten patients had a $4 \mathrm{~mm}$ fenestration placed in a tube of prosthetic material $(n=3)$ or in the prosthetic portion of a lateral tunnel $(n=7)$, or in the patch used to close an oval fossa defect $(n=1)$. In one of these patients, who had pulmonary atresia with an intact septum, persisting cyanosis after successful occlusion of the fenestration was due to shunting through a second tricuspid valve orifice, which communicated between the right atrium and left ventricle. This patient subsequently had a second procedure to occlude this atrioventricular communication (fig 1). In one infant a fenestration was placed in the patched atrial septum to decompress the left atrium after closure of a primum atrial septal defect in the presence of a hypoplastic left ventricle. The left ventricle increased in size but there was a large left to right shunt across the stretched defect $(3 \cdot 2: 1)$ and so closure of this fenestrated pericardial patch was attempted 11 weeks after the original operation.

There were four patients with naturally occurring oval fossa defects. In one patient (the oldest) there was a substantial right to left shunt (resting oxygen saturation $86 \%$, falling to $64 \%$ on exercise) late after repair of a common arterial trunk. There were normal right sided pressures in this patient but evidence of a restrictive right ventricle on Doppler studies. The three smallest patients all had a naturally occurring secundum atrial septal defect with significant left to right shunt. There were two with coexistent bron- 

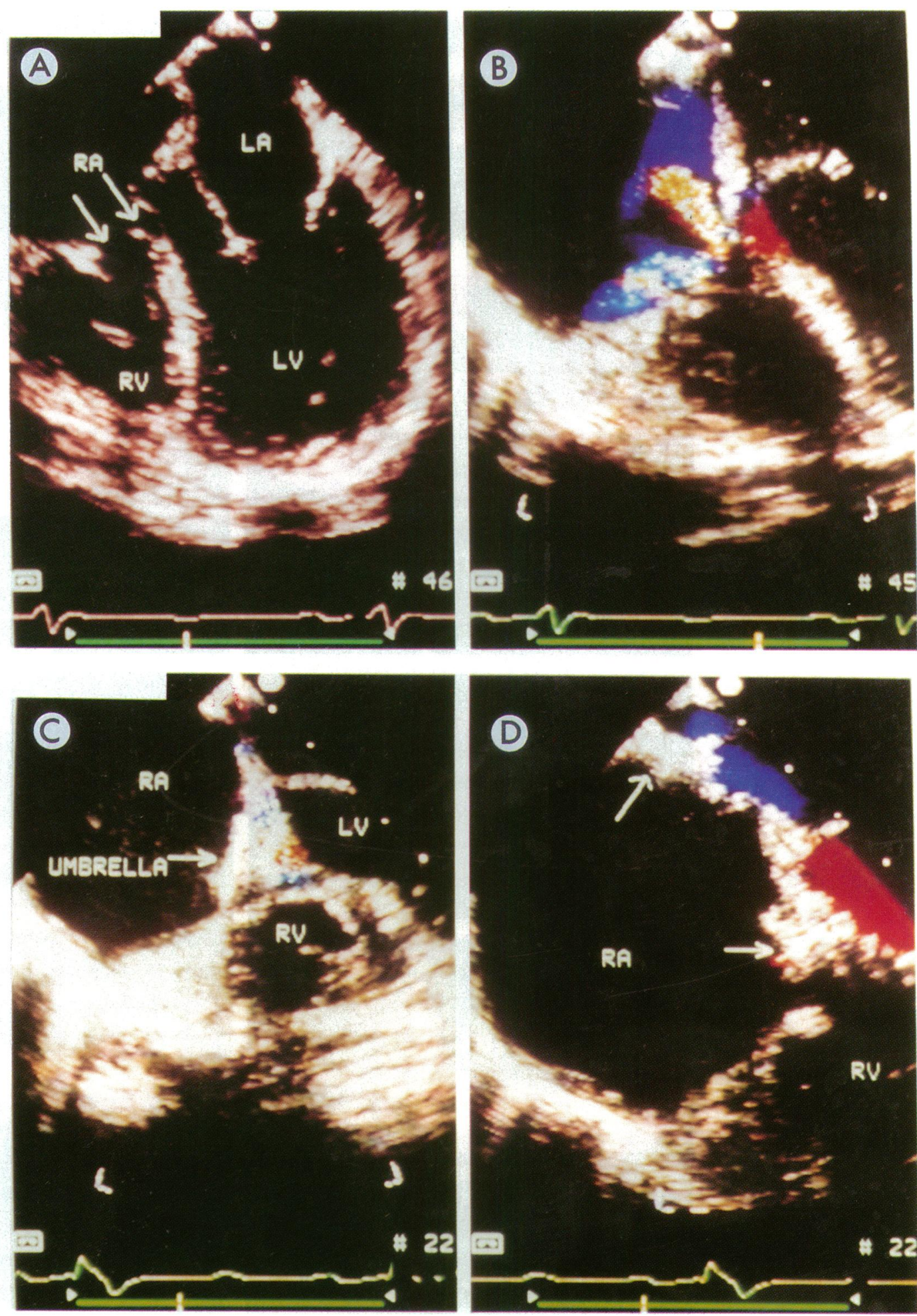

Figure 1 (A) Diastolic frame of a transoesophageal echocardiogram showing accessory tricuspid valve orifice connecting the right atrium to the left ventricle after fenestrated Fontan operation. The other orifice connects to the diminutive right ventricle in the usual way (see text for details). (B) Systolic frame showing left ventricular to right atrial shunt through the accessory orifice and right ventricular to right atrial shunt through to normal orifice. (C) The umbrella has been placed in the accessory orifice. There is trivial systolic left ventricular to right atrial shunting on colour flow mapping. (D) The two umbrellas in place (arrows).

chopulmonary dysplasia after neonatal ventilation for hyaline membrane disease. One patient underwent umbrella closure for a persisting large left to right shunt after surgical repair of critical aortic valve stenosis.

METHODS

The umbrella was modified in each case by the methods described previously. ${ }^{4}$ All but two of the umbrellas used were of the larger $17 \mathrm{~mm}$ size. The modified device was loaded and placed by the standard technique developed for closure of the arterial duct in all but one of the cases; one $17 \mathrm{~mm}$ device was front loaded into a 9 French $(F)$ sheath with the modification described by Perry and Lock. ${ }^{5}$ Transcatheter closure of the communication was only attempted in those patients in whom 

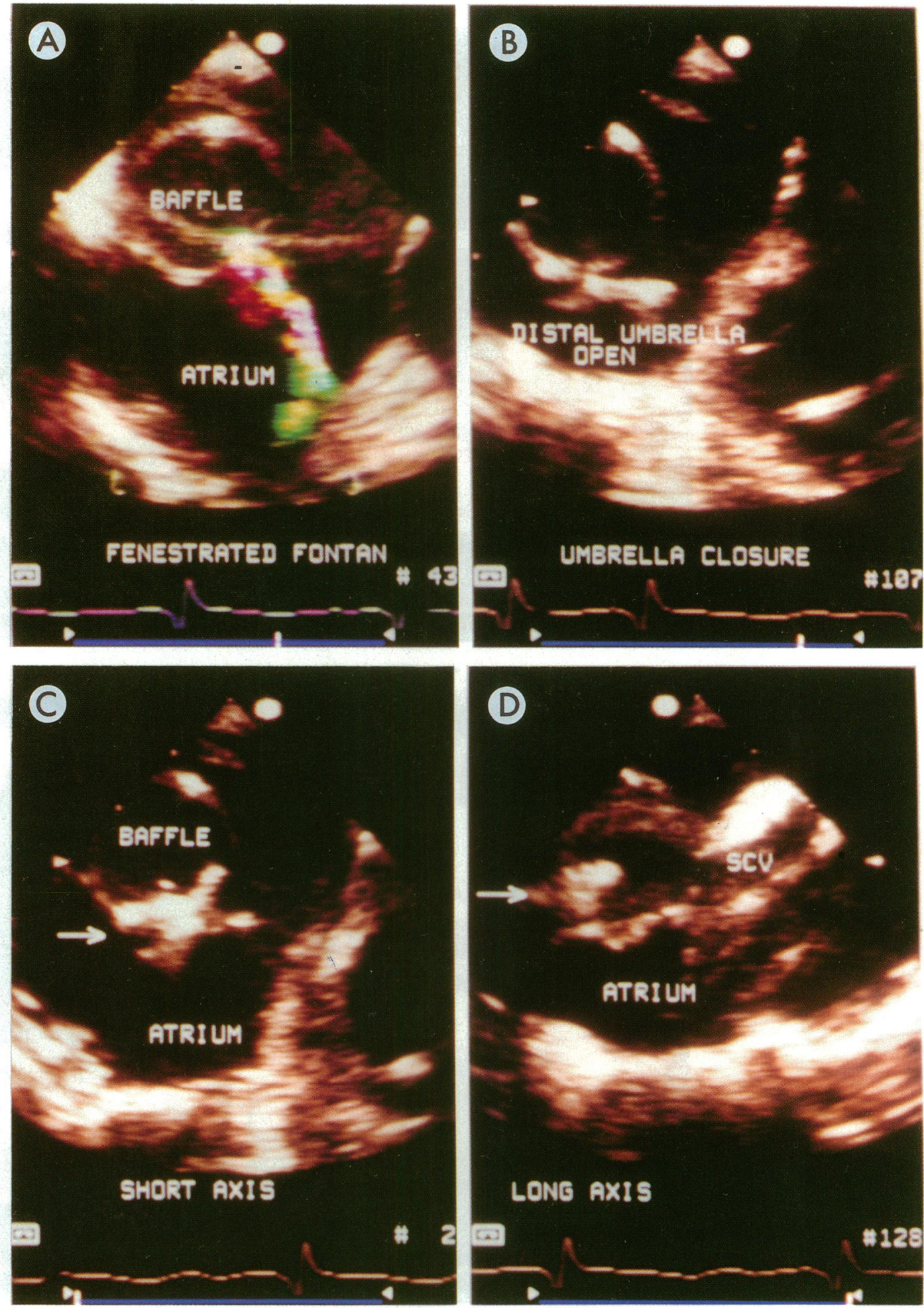

Figure 2 Transoesophageal echocardiograms. (A) Colour flow map showing $4 \mathrm{~mm}$ fenestration within intra-atrial baffle after the fenestrated Fontan operation. There is a wire traversing the fenestration. (B) The distal umbrella is open and being moved toward to the fenestration. (C) Vertical and (D) horizontal sections, showing position of the umbrella with the proximal umbrella open.

balloon sizes suggested a stretched diameter of $<8 \mathrm{~mm}$, and when balloon occlusion showed satisfactory haemodynamics in the case of the fenestrated Fontan operation. Placement was guided by simultaneous transoesophageal Doppler echocardiography (Hewlett Packard adult and paediatric probes) in all patients. All patients received procedural heparinisation of $50 \mathrm{IU} / \mathrm{kg}$ at the beginning of the study and $50 \mathrm{IU} / \mathrm{kg}$ every 30 minutes thereafter. All of our patients now receive formal anticoagulation with warfarin after the Fontan operation and this was continued after umbrella closure of the fenestration. All patients underwent immediate postoperative haemodynamic assessment with transoesophageal Doppler echocardiography. On follow up, transthoracic Doppler echocardiography was performed every six months. A routine chest radiograph was examined for 


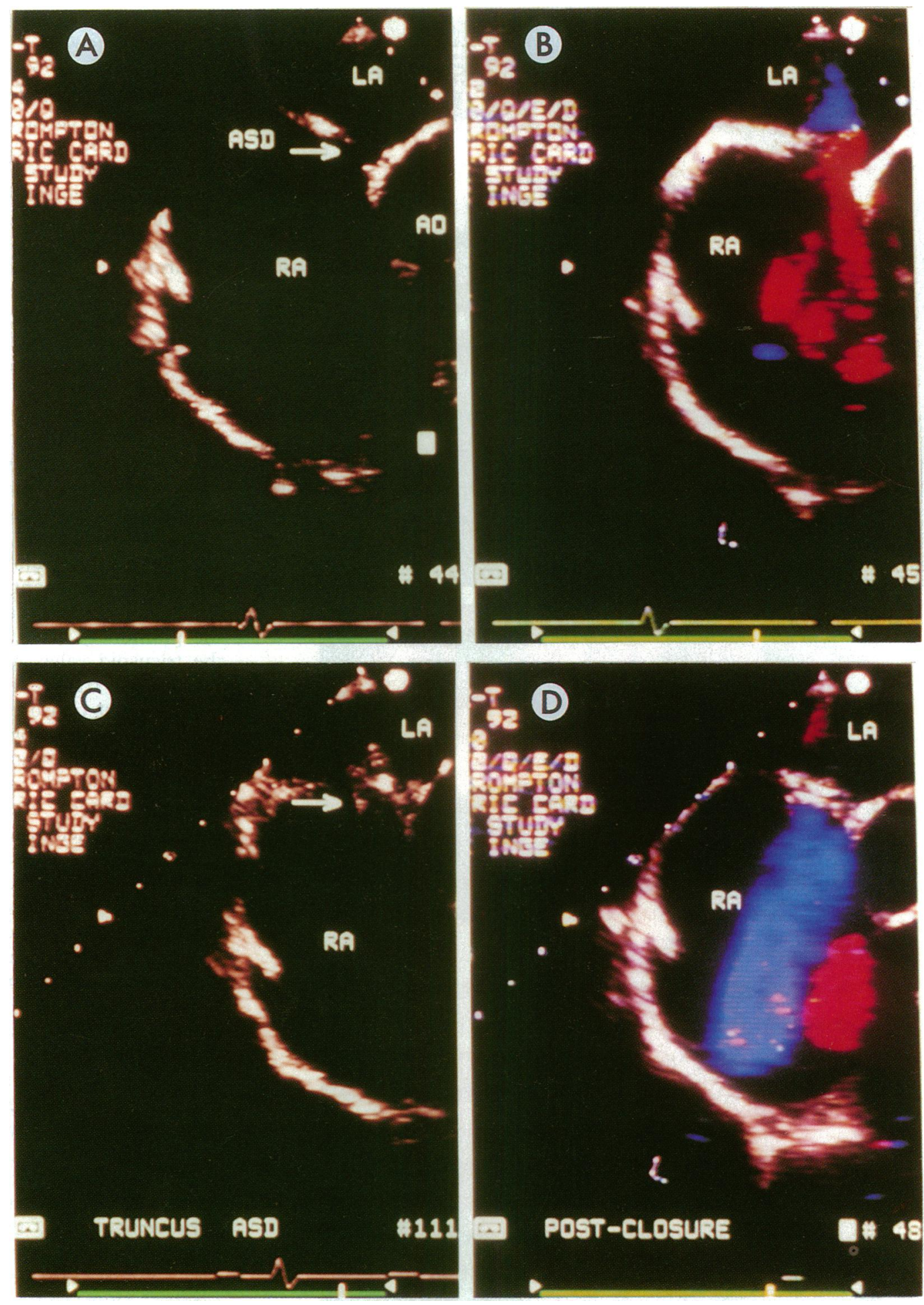

Figure 3 Transoesophageal echocardiograms $(A) A$ secundum atrial septal defect after repair of common arterial trunk 10 years previously. (B) A colour flow map illustrating the right to left atrial shunt through the defect. (C) Placement of distal umbrella into the defect (marked with arrow). (D) The device after release within the defect. Note the close apposition of the two umbrellas after modification.

possible umbrella arm fractures on each occasion. One patient has undergone cardiac catheterisation for other reasons.

\section{Results}

There were two procedural failures, both in patients after the fenestrated Fontan operation. In one there was complete occlusion of the inferior caval vein and because of the anatomy of a previously performed bidirectional Glenn procedure it was not possible to obtain a stable position across the fenestration with an internal jugular approach. In another patient there was femoral venous or inferior vena caval spasm. It was impossible to advance the $11 \mathrm{~F}$ sheath, and so the procedure was abandoned.

RIGHT TO LEFT SHUNT (11 UMBRELLAS IN 10 PATIENTS)

This group included the nine patients after fenestrated Fontan operation (fig 2) and the patient with a naturally occurring secundum 

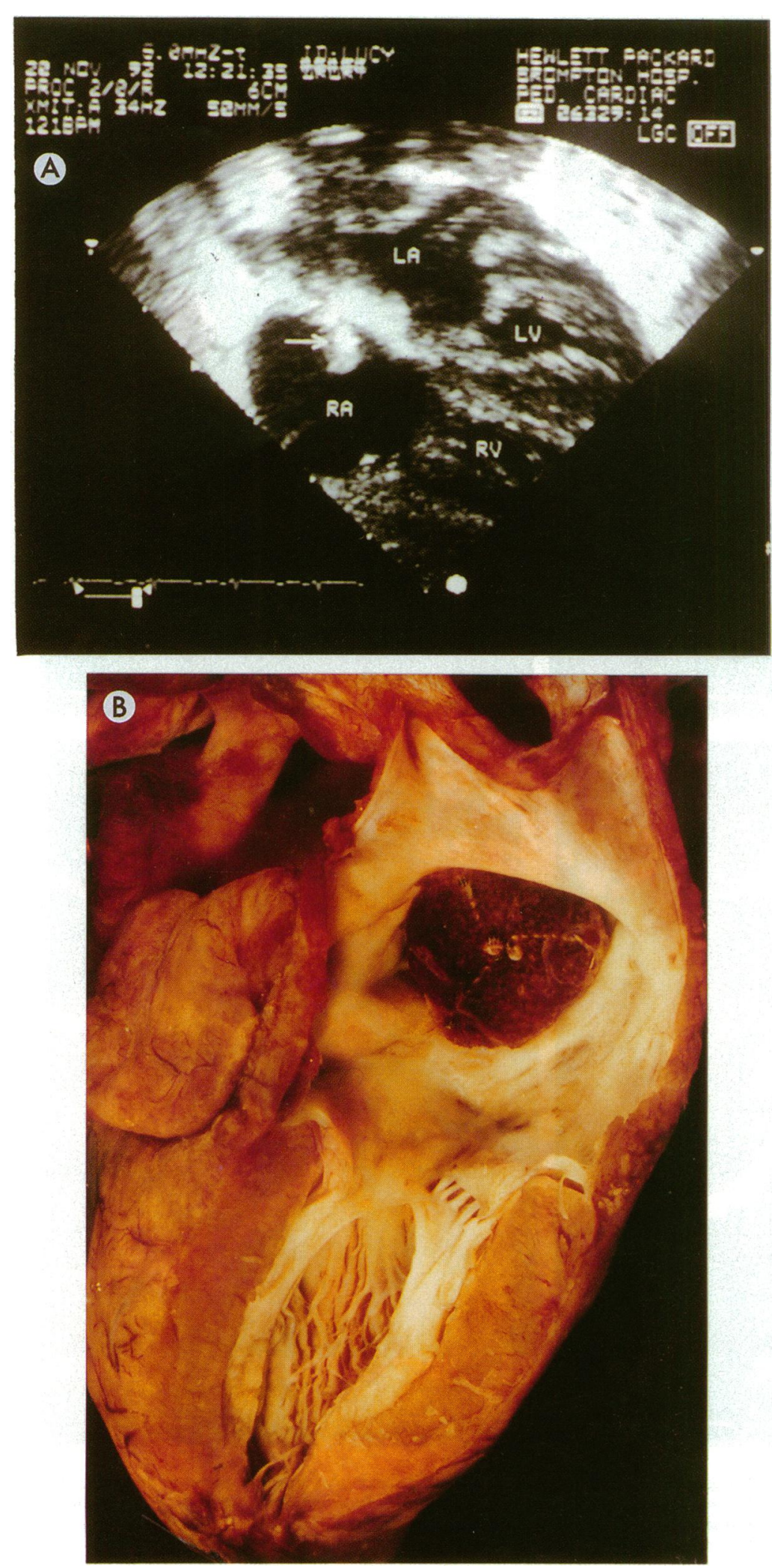

Figure 4 (A) Transoesophageal subgastric four chamber view of a $12 \mathrm{~mm}$ umbrella being placed in the atrial septum. The arrow marks the tip of the guiding sheath. (B) Necropsy specimen from the same patient as in $(A)$. This patient died from tamponade after removal of a left atrial line. The left atrial umbrella of this $12 \mathrm{~mm}$ device can be seen within the atrial septum, and totally occluding the atrial septal defect.

atrial septal defect with a right to left shunt late after repair of a common arterial trunk (fig 3). Transcatheter placement of a modified $17 \mathrm{~mm}$ umbrella was successful in all of these patients. There were no procedural complications. The preoperative arterial oxy- gen saturation in air rose from $87 \%(6 \%)$ to 93\% (3\%) after the procedure $(P<0.01)$.

Follow up is now six to 23 months. Transthoracic Doppler echocardiograms confirm complete closure of the defect in all but one patient. In this patient there was a trivial residual right to left shunt through the accessory atrioventricular valve orifice in which a $17 \mathrm{~mm}$ device had been placed. This patient also underwent cardiac catheterisation that confirmed a trivial right to left shunt in diastole and left to right shunt in systole. The resting arterial oxygen saturation in this patient was $96 \%$. No umbrella arm fractures have been shown on static radiographic screening.

LEFT TO RIGHT SHUNT ( $=4)$

There were two patients in whom unsatisfactory placement of the umbrella led to early surgery. In one patient with bronchopulmonary dysplasia the $17 \mathrm{~mm}$ device embolised to the pulmonary artery within one minute of placement while the patient was still under anaesthesia. It was retrieved from the pulmonary artery but became caught in the tricuspid valve apparatus and could not be removed transvenously with safety. In the other patient (closure of fenestrated primum atrial septal defect) one of the distal arms of a $12 \mathrm{~mm}$ umbrella was seen to prolapse on to the right atrial side of the defect. Because of this the patient underwent uncomplicated closure of the fenestration and removal of the umbrella device. Successful closure of a secundum atrial septum defect was achieved in two patients in this group. A three month old patient with bronchopulmonary dysplasia and a left to right shunt of $2 \cdot 5: 1$ had complete closure with a modified $17 \mathrm{~mm}$ device. Finally, a 5 day old patient who had undergone successful surgical relief of critical aortic stenosis had a low cardiac output in the immediate postoperative period. At subsequent cardiac catheterisation a 3.2:1 left to right shunt was shown across a secundum atrial. septal defect or stretched foramen ovale. Successful closure with a modified $12 \mathrm{~mm}$ device led to a dramatic clinical improvement and the patient was subsequently removed from inotropic and ventilatory support. He died five days later from cardiac tamponade after removal of an indwelling left atrial line placed at the time of the original surgery. At necropsy, the atrial septal defect was completely occluded by the $12 \mathrm{~mm}$ device (fig 4).

\section{Discussion}

Our study shows that it is possible to close interatrial communications with a modified Rashkind umbrella device. Although not specifically designed for this purpose, we believe that the clinical benefit obtained by its use outweighs the potential disadvantages in these carefully selected cases.

The fenestrated Fontan operation has now been shown conclusively to decrease the morbidity and possibly the mortality of high risk 
Fontan operations. ${ }^{2}$ By deliberately leaving a residual interatrial communication systemic cardiac output is maintained at the expense of persisting cyanosis. Subsequent elective clamshell closure is successful in restoring a normal systemic arterial saturation, and improved oxygen delivery in those patients in whom test occlusion yields a satisfactory haemodynamic result. We have shown that it is possible to use a modified $17 \mathrm{~mm}$ patent ductus arteriosus umbrella device to the same effect. By placing a bend in each of the arms of the distal and proximal umbrellas it is possible to obtain circumferential apposition so that adequate closure of defects in the patched atrial septum or artificial material of an intra-atrial tube or baffle can be achieved. Indeed, there were no adverse sequelae in our patients. Furthermore, there were no umbrella arm fractures found during six to 23 months follow up. There was only one patient with a naturally occurring atrial septal defect in this group. Although small $(8 \mathrm{~mm}$ in diameter) there was a significant right to left shunt across it and successful occlusion restored normal resting and exercise arterial saturations with considerable clinical improvement. Finally, we attempted to close a secondary atrioventricular valve orifice, that was allowing a persistent right atrial to left ventricular shunt in one of the patients who had previously undergone a fenestrated Fontan operation. Technically this was uncomplicated, but a residual diastolic right to left and systolic left to right shunt persists 18 months after umbrella placement. None the less, there is only minimal arterial desaturation and no significant left to right shunt was found on oxymetry. All of these patients have avoided surgery, and the benefits of umbrella closure of their defects are obvious.

We were less successful in our group with left to right shunts. There were two important complications. In one there was unexplained embolisation a minute or so after placement, and in the other there was prolapse through the fenestration of one of the arms of the umbrella placed in a pericardial patch to close a primum atrial septal defect in a patient with a hypoplastic left ventricle. At operation, both defects were found to be eccentric, and although the stretched diameter and transoesophageal echocardiogram seemed to be satisfactory at the time of cardiac catheterisation the longest axis in the unstretched state was subsequently shown to be greater than that acceptable for the selected device. Both of the defects were borderline in size and we think that the eccentric nature of the defect allowed prolapse of the device through it and led to these complications. Both patients had uncomplicated surgery, and there were no long lasting ill effects.

There were two patients in whom successful occlusion of secundum atrial septal defect with left to right shunts was achieved. Both of these patients had immediate clinical improvement although one subsequently died due to cardiac tamponade after removal of an indwelling left atrial cannula. At necropsy the device was shown to be completely occluding the atrial septal defect and partially endothelialised only five days after placement (fig 4).

Although representing our entire learning experience we think that these results justify the continued use of the modified umbrella device in symptomatic patients who need surgical closure of a naturally occurring atrial septal defect, or in patients after the fenestrated Fontan operation. We think that this device is not yet suitable for routine closure of secundum atrial septal defects in asymptomatic patients, although it should be possible to expand the indications for its use if a larger device of similar design were available.

We thank our surgical and pathology colleagues for their sup port. We thank Dr Elliot Shinebourne and Dr Jane Somerville for allowing us to study their patients.

1 Rome JJ, Keane JF, Perry SB, Spevak PJ, Lock JE Double-umbrella closure of atrial defects: initial clinica applications. Circulation 1990;82:751-8.

2 Bridges ND, Lock JE, Castandea AR. Baffle fenestration with subsequent transcatheter closure. Circulation 1990 82:1681-9.

3 Bridges ND, Perry SB, Keane JF, et al. Preoperative transcatheter closure of congenital muscular ventricular septal defects. N Engl ₹ Med 1991;324:1312-7.

4 Redington AN, Rigby ML. Novel uses of the Rashkind ductal umbrella in adults and children with congenital ductal umbrella in adults and children
heart disease. $B r$ Heart $\mathcal{f}$ 1993;69:47-51.

5 Perry S, Lock J. Front-loading of double-umbrella devices, a new technique for umbrella delivery for closing cardiovascular defects. Am f Cardiol 1992;70 917-20. 